\title{
Automated Multichamber Time-lapse Videography for Long-term In Vivo Observation of Migrating Cells
}

\author{
HELMUT BUHLER $^{1}$, RAPHAEL ADAMIETZ ${ }^{1}$, THERESA ABELN ${ }^{1}$, DAVID DIAZ-CARBALLO ${ }^{2}$, \\ PASCALINE NGUEMGO-KOUAM ${ }^{1}$, THOMAS HERO $^{3}$ and IRENAUS A. ADAMIETZ ${ }^{3}$ \\ ${ }^{1}$ Institute for Molecular Oncology, Radiobiology and Experimental Radiotherapy, \\ Marien Hospital Herne, Medical Center Ruhr-University Bochum, Herne, Germany; \\ ${ }^{2}$ Clinic for Hematology and Oncology, Medical Center Ruhr-University Bochum, Bochum, Germany; \\ ${ }^{3}$ Clinic for Radiotherapy and Radio-Oncology, Medical Center Ruhr-University Bochum, Bochum, Germany
}

\begin{abstract}
Aim: To observe and document the migration of living cells by time-lapse videography, we constructed a lowbudget system based on a common inverted microscope. Materials and Methods: Long-term observation of six-well plates is enabled through maintenance of cell culture conditions $\left(5 \% \mathrm{CO}_{2}\right.$ in air at $\left.37^{\circ} \mathrm{C}\right)$. Points of interest can be revisited in definable intervals with $<1 \mu \mathrm{m}$ repositioning error. Digital photographs from each programmed time point are paired with environmental data and combined into a record. Results: We used this new chamber to observe the migration of various cell lines. The design represents a good compromise between low cost and good precision. Detailed analyses verified that the environmental conditions were appropriately maintained, enabling long-term observation of viable cells. The stimulating influence of irradiation with photons (radiotherapy) on cellular motility of glioblastoma cells is presented. Conclusion: This study demonstrates that useful videographic systems can be constructed at low cost.
\end{abstract}

Although videography is a critical tool for studies of behavior of living cells, commercially available videographic systems incur high investment costs and often cannot be flexibly adjusted to meet experimental requirements. 'Homemade' environmental chambers have been documented since the early 1950s (1-7) with various chamber types, many of which closely resemble modern designs (8-11). More recently, environmental chambers have been combined with

This article is freely accessible online.

Correspondence to: Dr. Helmut Bühler, Universitätsklinikum Marien Hospital, Hoelkeskampring 40, D-44625 Herne, Germany. Tel: +49 23234991058, Fax: +49 23234991059, helmut.buehler@rub.de

Key Words: Time-lapse videography, environmental chamber, live cell imaging, migrating cells, cellular motility. time-lapse videography to study living cells (12-16). The basic demands on such a chamber include maintenance of temperature, humidity, and atmosphere (generally $5 \% \mathrm{CO}_{2}$ in air), as well as precise and reproducible chamber positioning in order to simultaneously observe selected areas. The environmental chamber should contain at least two separate compartments, to ensure that control samples can be manipulated and observed alongside the experimental samples; standard multiwell plates are flexible, simple to handle, and low-cost.

We wished to demonstrate that it is possible to construct a low-cost device to meet these various experimental requirements. A thermostatically controlled plate holder enclosed by a small transparent casing with maintained gas flow was selected as the optimal design.

\section{Materials and Methods}

We started with a second-hand inverted fluorescence microscope (Axiovert 25; Zeiss, Oberkochen, Germany), which we mounted onto a plate that served for mechanical fixation and static positioning of the components (Figure 1A). A servo motor was connected to the manual drive of the microscope to automate objective focusing. In addition, two linear servo motors (Linax Lxc 44F04; Jenny Science, Rain LU, Switzerland) were installed to allow planar positioning of the plate holder, with positioning repeatability of $\pm 5 \mu \mathrm{m}$ (manufacturer's data); we recorded an accuracy of reposition $<1 \mu \mathrm{m}$ (obtained from comparison of consecutive images, data not shown). This setup permits reproducible spatial positioning to focus on freely selectable points of interest on the multiwell plate.

The microscope was equipped with a second optical path and a connector for cameras. A digital SLR camera was mounted to the microscope for documentation of imaging.

In our experimental setting, the cells require a constant temperature of $37^{\circ} \mathrm{C}$ and a carbonate-buffered culture medium at $5 \%$ $\mathrm{CO}_{2}$ in air saturated with water. The microscope was designed to house standard six-well plates for cell culture, for reasons of low cost, simplicity, and reproducibility. To avoid microscope corrosion, only the multiwell plate was enclosed, requiring transparent housing. 

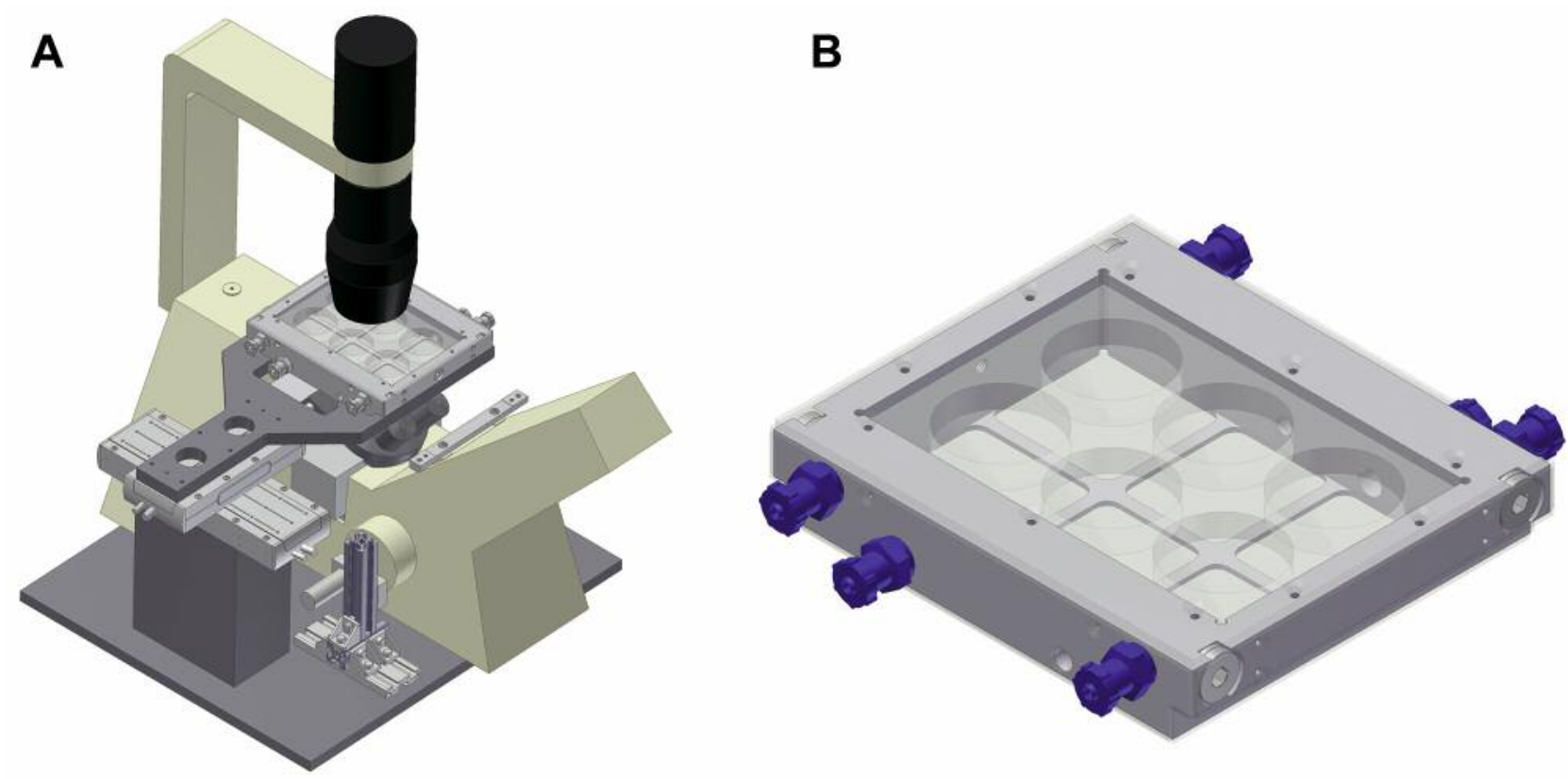

C

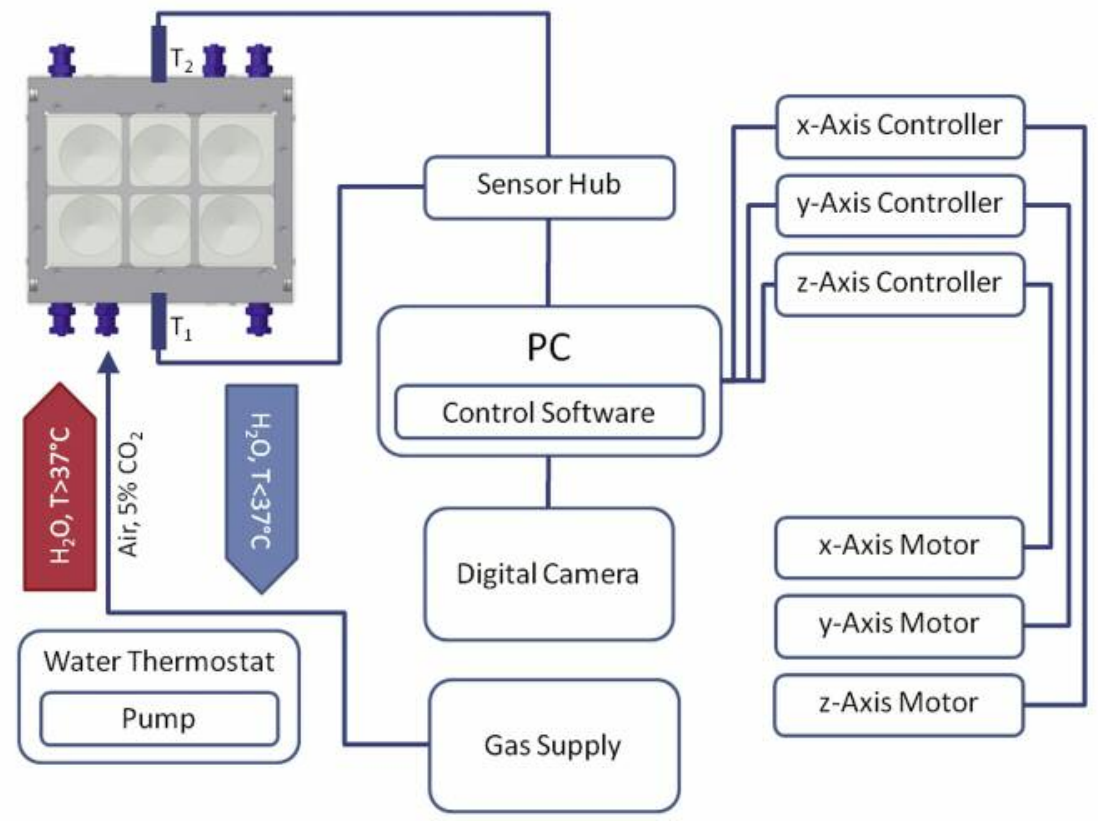

Figure 1. Assembly of the videographic system. A: The system is based on a Zeiss Axiovert 25 microscope. A plate holder for standard multiwell plates is positioned precisely by two linear motors; reproducible focusing of points of interest is performed by a servo motor mounted to the manual drive of the microscope. B: The receptacle is designed to hold standard multiwell plates. The housing is sealed by transparent plates, and a constant atmosphere is secured by flooding the housing with $5 \% \mathrm{CO}_{2}$ in air. Temperature is maintained by a water circuit in the aluminum frame. C: The system layout. The flowchart shows how the plate holder is controlled for data collection and how the environmental conditions are maintained.

The plate holder is based on a cuboid design with a rectangular pocket for the multiwell plate, which is passively positioned by a spring mechanism (Figure 1B). The bottom of the pocket contains a grating of narrow bars to conduct heat. Two channels in the aluminum frame (chosen for its high thermal conductivity) allow water to flow through, thereby controlling the temperature, and two gas connectors maintain the atmosphere in the housing. The entire apparatus is housed with transparent polycarbonate, chosen for its low thermal conductivity. Two sensors were installed to monitor temperature during the course of experiments. 
The system layout includes a thermostat with an attached pump (Thermostat 2761; Eppendorf, Hamburg, Germany) to supply the plate holder with circulating heated water to maintain the temperature at $37^{\circ} \mathrm{C}$ (Figure $1 \mathrm{C}$ ). A dose-controlled $\mathrm{CO}_{2}$ dispenser was excluded from the system layout to minimize costs. A commercially available gas mixture of $5 \% \mathrm{CO}_{2}$ in synthetic air (Carblood 5; Air Liquide, Düsseldorf, Germany) was humidified by slowly bubbling it through water, then piping it through the plate holder.

The temperature sensors, digital camera (Olympus E-420), and axis controllers were connected to a personal computer. We developed custom software named MicroControl to collect sensor data and to control the axes and digital camera. The camera provides a live picture feed to the program; the user can position the plate from the live view and define points of interest. Parameters such as time interval and end of run can also be set. The plate holder automatically moves to the user-defined points, where a photograph is taken and automatically downloaded to a predefined folder. (Interested individual may contact us to obtain a free copy of the software). After the full set of cycles is completed, the entire set of images can be uploaded to a commercial video program (Pinnacle Video Studio 10, Corel GmbH, München, Germany) and assembled into a video sequence. The motility of observed cells can be analyzed using freely available software. We tracked the cells with ImageJ (download from the NIH homepage, rsbweb.nih.gov/ij/download.html). The motility parameters velocity, accumulated, and Euclidean distance were analyzed with the ibidi Chemotaxis and Migration Tool (free download from the ibidi homepage ibidi.com/software/chemotaxis_and_migration_tool/).

\section{Results}

We assayed the reliability and utility of our automated multichamber time-lapse videographic system for the following requirements: maintenance of temperature of the culture medium over a sustained period of time; maintenance of humidity near $100 \%$ to avoid osmolality changes through evaporation; maintenance of $5 \% \mathrm{CO}_{2}$ in air; generation of video-quality digital images; and precision of culture plate repositioning for unambiguous identification of cells of interest.

The temperature of the plate holder was continuously monitored by a PT-sensor located in the aluminum frame; values were paired and saved with every image. The measured temperature exhibited a deviation of $<1^{\circ} \mathrm{C}$ within $24 \mathrm{~h}$; over 10 runs, the minimal and maximal values were $36.2^{\circ} \mathrm{C}$ and $37.7^{\circ} \mathrm{C}$, respectively (Figure 2).

To assay humidity stability, the plate was weighed immediately before and after the run to detect loss of medium from evaporation causing an increase in osmolality. We detected marginal weight loss over six experiments; the mean starting value ( \pm standard error of the mean) was $70.66 \mathrm{~g}( \pm 0.06 \mathrm{~g})$, and the mean end value was $70.32 \mathrm{~g} \pm(0.08 \mathrm{~g})$.

Standard cell culture media contain phenol red as a $\mathrm{pH}$ indicator. The basic form of phenol red shows strong absorption at $558 \mathrm{~nm}$ in contrast to the protonated molecule, with maximal absorption at $430 \mathrm{~nm}$. Therefore, the difference in absorption at these two wavelengths is a very sensitive measure reflecting the

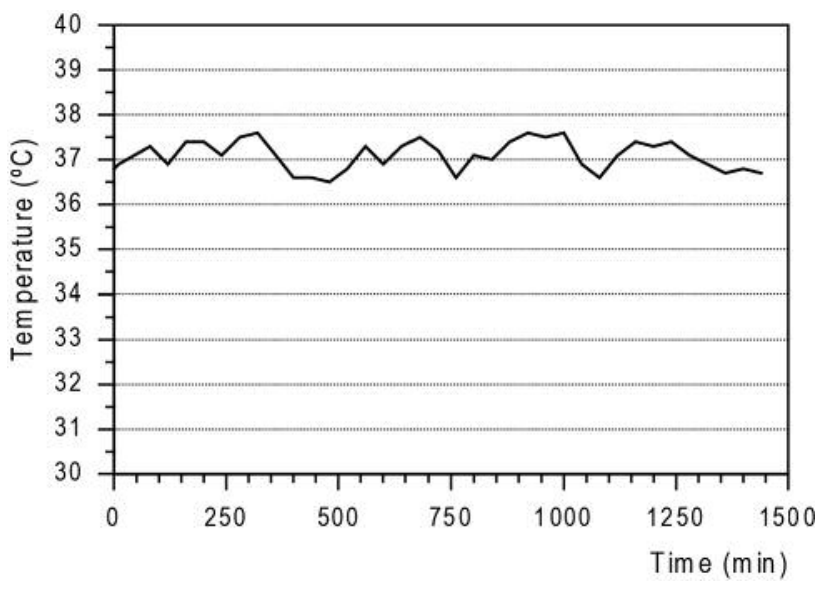

Figure 2. The course of temperature during a single run. Temperature maintenance is achieved through the connected thermostat set to $37.0^{\circ} \mathrm{C}$. The temperature of the plate holder was continuously monitored by a PT-sensor located in the aluminum frame; values were paired and saved with every image over a period of $24 \mathrm{~h}$.

$\mathrm{pH}$ of the medium. We collected the absorbance values at 450 and $570 \mathrm{~nm}$ with an ELISA reader (Multiskan Ascent, Labsystems, Germany) to monitor changes in $\mathrm{pH}$. We observed a continuous increase in $\Delta$ absorbance $(570-450 \mathrm{~nm})$ with an asymptotic approach to an upper limit when the plate was brought from $5 \% \mathrm{CO}_{2}$ to room conditions. This limit at total loss of dissolved $\mathrm{CO}_{2}$ is slowly reached within $2 \mathrm{~h}$ so that a rapid measurement after the run yields useful data (Figure 3A). In order to monitor $\mathrm{CO}_{2}$ and $\mathrm{pH}$ levels in the videographic system, the plates were transferred immediately before and after the run to the ELISA reader to determine absorption values at $570 \mathrm{~nm}$ and $450 \mathrm{~nm}$. Six independent experiments with starting $\mathrm{pH}$ levels between 7.2 and 7.3 exhibited ending values between 7.2 and 7.5, a marginal change during the course of the experiment (Figure 3B).

Figure 4 shows a typical photograph taken by the system with a Zeiss LD A-Plan $\times 10$ objective. The quality is not of highest resolution but is good enough for the observation of migrating cells. We were able to document changes in the organization of filipodiae and moving fronts.

Figure 5 demonstrates live cell imaging using this system. In order to determine the influence of low-dose irradiation on the migration of U373 human glioblastoma cells, a single dose of 0.5 Gy photons from a linear accelerator (Synergy S, Elekta, Hamburg, Germany) was applied. The motility of irradiated and non-irradiated cells (as controls) was observed and documented with the videographic system. The graph shows clearly that cellular motility is enhanced by low-dose irradiation. The Euclidean distance (the straight line between the origin and endpoint of travelling cells) in particular was increased markedly from $66.9 \pm 4.21$ to $97.4 \pm 6.90 \mu \mathrm{m}$ per day $(p<0.001)$. 

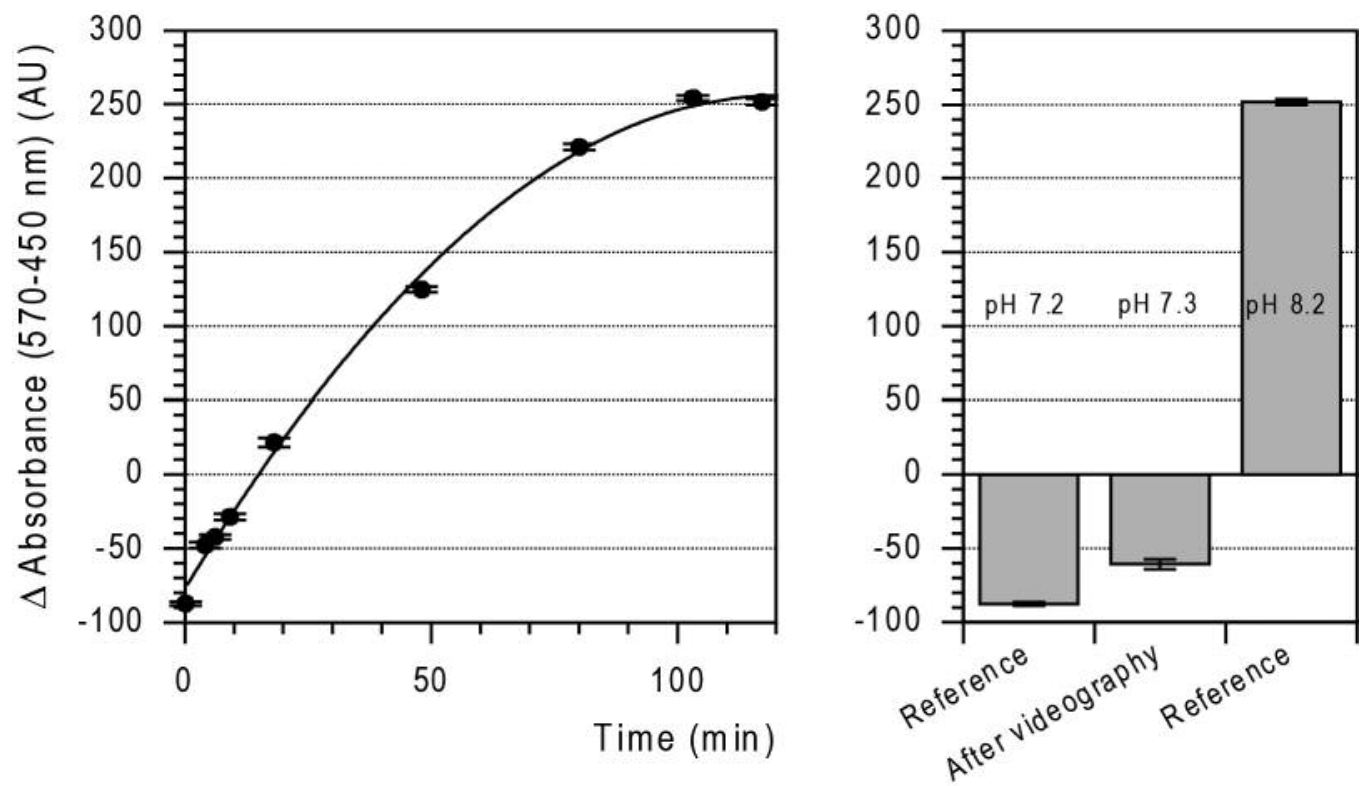

Figure 3. Monitoring pH levels via absorption maxima of phenol red in the cell culture medium. Acidic and basic peaks of the absorption spectrum were determined at 450 and $570 \mathrm{~nm}$ with an ELISA reader and their difference used as a measure for the pH value of the medium. A: Time course of the $\mathrm{pH}$ increase as a function of $\mathrm{CO}_{2}$ loss after plate transfer from $5 \% \mathrm{CO}_{2}$ in the incubator to room conditions. B: Mean absorption value of the plates immediately after the run compared to reference values at defined $\mathrm{pH}$. Data depicted are mean \pm standard error of the mean from six independent experiments.

\section{Discussion}

Important features of malignant cancer cells include motility and migration. Of special interest is cellular behavior in 'social' contexts that include the influence of neighboring cells in an in vivo-like arrangement of stroma, blood vessels and the immune system. These dynamic interactions require videographic documentation, but commercially available systems are very expensive and demand substantial investment costs. In addition, most commercial systems are unable to monitor multiple experiments in parallel.

In this article we describe the low-budget construction of a videographic system based on a Zeiss inverted fluorescence microscope. Environmental chambers for cell culture can be classified as closed or open systems; however, open systems are not amenable to long-term observation of viable cells because environmental control and sterility cannot be maintained. Closed systems, on the other hand, are usually expensive and difficult to handle.

Our system was able to document the migration of tumor cells; the analysis and evaluation of gathered data enabled the analysis of cellular behavior. The results were consistently reproduced in all cases, supporting the experimental value of our videographic system.

Our final platform design represents a good compromise between low cost and precision, satisfying the experimental

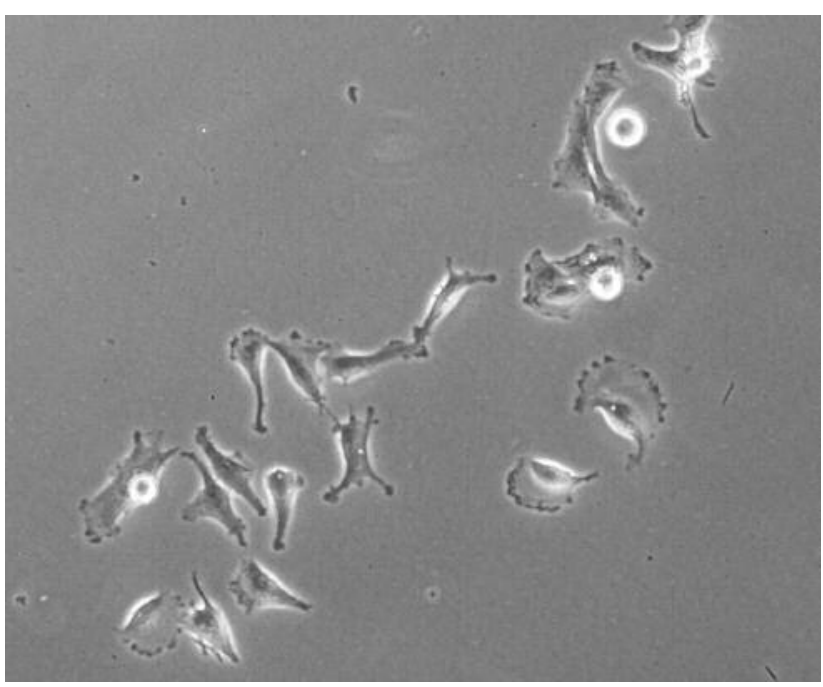

Figure 4. U87 glioblastoma cells after $62 \mathrm{~h}$ in the environmental chamber. One image out of a series of 72 consecutive photos taken by the videographic system is shown.

requirements of the proof-of-concept analyses provided here. The environmental conditions were appropriately maintained, enabling long-term observation of viable cells. We anticipate that this work will inspire future investigations through the low-cost videographic construction detailed here. 

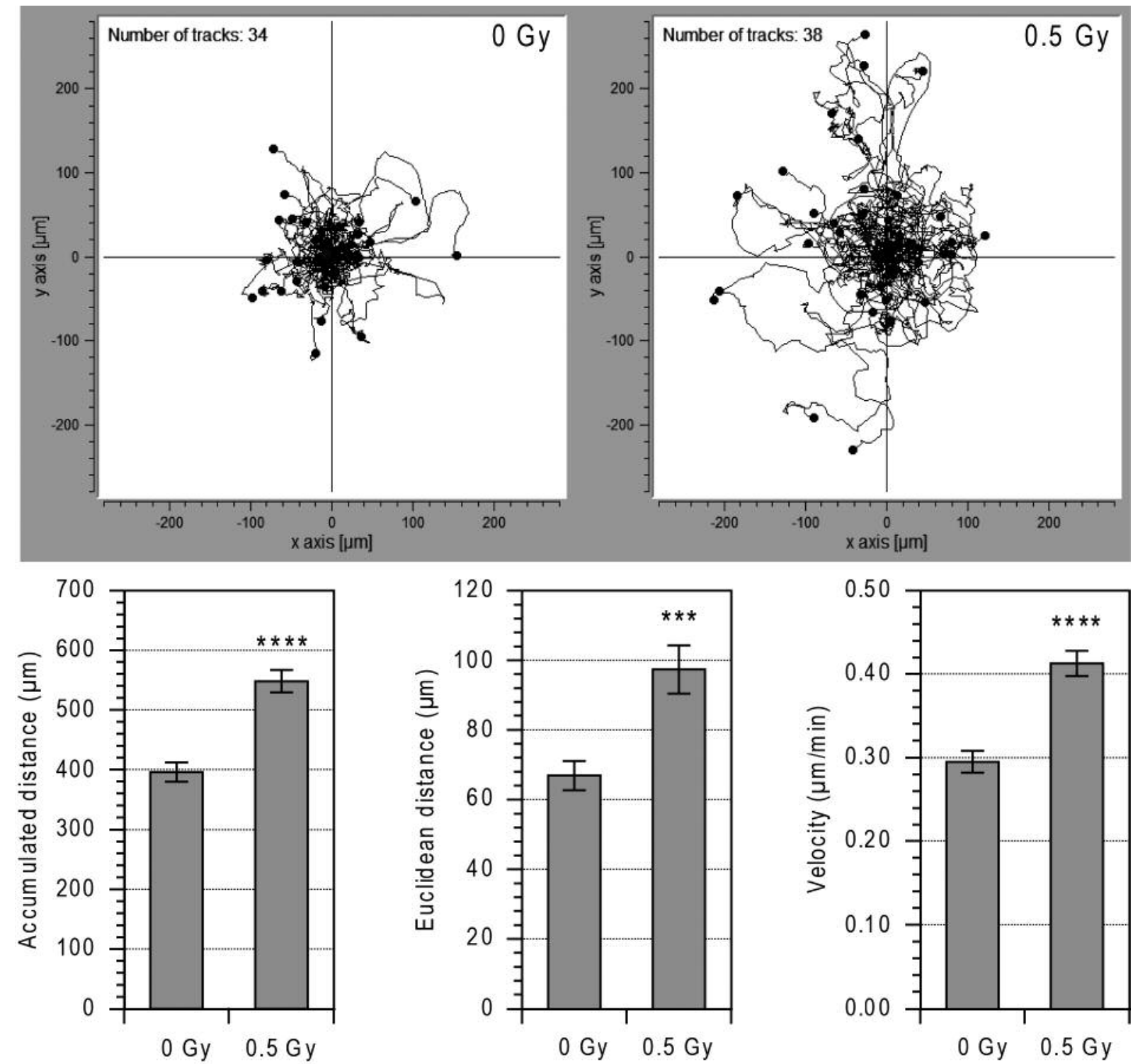

Figure 5. U373 glioblastoma cells were irradiated with 0.5 Gy photons. These cells and non-irradiated controls were transferred to the videographic system and observed over a period of $24 \mathrm{~h}$. Three areas of interest were selected for each sample and photographed every 10 min. Randomly selected cells were tracked with ImageJ and analyzed with the ibidi migration tool. Typical migration patterns for both samples are shown. The columns represent means \pm standard error of the mean of 90-100 travelling cells. The level of significance was analyzed with Student's $t$-test. $*^{* *} p<0.001$; **** $p<0.0001$.

\section{Acknowledgements}

The Authors would like to thank Anja Kochanneck and Bettina Priesch-Grzeszkowiak for excellent assistance and the Stiftungsfonds BIOX for supporting this work.

\section{References}

1 Bavister BD: A minichamber device for maintaining a constant carbon dioxide in air atmosphere during prolonged culture of cells on the stage of an inverted microscope. In Vitro Cell Dev Biol 24: 759-763, 1988 .
2 Christiansen GS, Danes B, Allen L and Leinfelder PJ: A culture chamber for the continuous biochemical and morphological study of living cells in tissue culture. Exp Cell Res 5: 10-15, 1953.

3 Denkers IA, Dragowska W, Jaggi B, Palcic B and Lansdorp PM: Time lapse video recordings of highly purified human hematopoietic progenitor cells in culture. Stem Cells 11: 243-248, 1993.

4 Hing WA, Poole CA, Jensen CG and Watson M: An integrated environmental perfusion chamber and heating system for long-term, high resolution imaging of living cells. J Microsc 199: 90-95, 2000.

5 Ince C, Ypey DL, Diesselhoff-Den Dulk MM, Visser JA, De Vos $\mathrm{A}$ and Van Furth R: Micro- $\mathrm{CO}_{2}$-incubator for use on a microscope. J Immunol Methods 27: 269-275, 1983. 
6 Rose GG: A separate and multipurpose tissue culture chamber. Tex Rep Biol Med 12: 1075-1081, 1954.

7 Rose GG, Kumegawa M and Cattoni M: The circumfusion system for multipurposeculture chambers. II: The protracted maintenance of differentiation of fetal and newborn mouse liver in vitro. J Cell Biol 39: 430-450, 1968.

8 Heidemann SR, Lamoureux $\mathrm{P}$, Ngo K, Reynolds $\mathrm{M}$ and Buxbaum RE: Open-dish incubator for live cell imaging with an inverted microscope. Biotechniques 35: 708-714, 716, 2003.

9 Ho CL, Mou TY, Chiang PS, Weng CL and Chow NH: Mini chamber system for long-term maintenance and observation of cultured cells. Biotechniques 38: 267-273, 2005.

10 Petronis S, Stangegaard M, Christensen CB and Dufva M: Transparent polymeric cell culture chip with integrated temperature control and uniform media perfusion. Biotechniques 40: 368-376, 2006.

11 Picard C, Hearnden V, Massignani M, Achouri S, Battaglia G MacNeil S and Donald A: A micro-incubator for cell and tissue imaging. Biotechniques 48: 135-138, 2010.

12 Hardarson T, Hanson C, Claesson M and Stenevi U: Time-lapse recordings of human corneal epithelial healing. Acta Ophthalmol Scand 82: 184-188, 2004.
13 Kulesa PM and Kasemeier - Kulesa JC: Construction of a heated incubation chamber around a microscope stage for time-lapse imaging. CSH Protoc. pdb.prot4792. doi: 10.1101/pdb.prot4792, 2007.

14 Kwak YH, Hong SM and Park SS: A single cell tracking system in real-time. Cell Immunol 265: 44-49, 2010.

15 Orhan G, Baron S, Norozi K, Männer J, Hornung O, Blume H, Misske J, Heimann B, Wessel A and Yelbuz TM: Construction and establishment of a new environmental chamber to study realtime cardiac development. Microsc Microanal 13: 204-210, 2007.

16 Salierno M, Cabrera R, Filevich O and Etchenique R: Encapsulated Petri dish system for single-cell drug delivery and long-term time lapse microscopy. Anal Biochem 371: 208-214, 2007.

Received January 5, 2017

Revised March 28, 2017

Accepted March 31, 2017 